\title{
Sex hormones as an emerging weapon to combat COVID-19
}

Qasim Mehmood ${ }^{1}$, Priyanka Chahal ${ }^{2}$, Parth Patel $^{3}$, Prashant Upadhyay ${ }^{4}$, Arisha Nawaz ${ }^{5}$, Debraj

Mukhopadhyay $^{6}$, Anna Chiara Corriero ${ }^{7}$, Dattatreya Mukherjee* ${ }^{*}$, Usman Garba Kurmi ${ }^{9}$, Hadiza Abubakar Balkore $^{10}$, Vikash Jaiswal ${ }^{11}$

1. Faculty of Medicine, Undergraduate Research Scholar, King Edward Medical University, Lahore, Pakistan

2. Faculty of Medicine, Undergraduate Research Scholar, S. Tentishev Asian Medical Institute, Kyrgyzstan

3. Pharmaceutical Science Scholar, H. K. College of Pharmacy, Jogeshwari West, Mumbai400102, Maharashtra, India

4. Faculty of Medicine, Undergraduate Research Scholar, Government Medical College Jalaun, India,

5. Faculty of Medicine, Undergraduate Research Scholar, Dow International Medical College, Karachi, Pakistan

6. Public Health Scholar, School of Allied Health Sciences, Delhi Pharmaceutical Sciences \& Research University, New Delhi, India

7. Faculty of Medicine, Undergraduate Research Scholar, Anglia Ruskin University, United Kingdom

8. Faculty of Medicine, Undergraduate Research Scholar and Research Assistant, Jinan University, P.R China

9. Faculty of Science, Dept of Biochemistry, University of Maiduguri, Nigeria

10. Faculty of Science, Dept of Biochemistry, University of Maiduguri, Nigeria

11. Faculty of Medicine, Research Scholar, Larkin Community Hospital, Miami, United States of America

*Corresponding Author: Dattatreya Mukherjee, Faculty of Medicine and Undergraduate Research Scholar, Jinan University, P.R China, email: dattatreyamukherjee4u@ outlook.com

\section{Introduction}

Coronavirus disease 2019 (COVID-19) started as an epidemic in Wuhan in 2019 and was declared pandemic by WHO in March 2020. The virus has been identified and named as severe acute respiratory syndrome coronavirus 2 (SARS-CoV-2). This novel coronavirus strain is the causative agent of COVID-19, and continues to rapidly spread worldwide. SARS-CoV-2 is a highly pathogenic and transmissible coronavirus that spreads through respiratory droplets and unprotected close contact.(1) 
COVID-19 outbreak, which has caused $>95$ millions confirmed infections and $>2$ million coronavirus related deaths, is one of the most disastrous worldwide crises in recent years. Several methods have been used to examine SARS-CoV-2 infections. i.e. RT-qPCR for viral RNA detection, and rapid screening procedures for antibody or virus detection. COVID-19 shows an incubation period of 3-7 days globally. Approximately $80 \%$ of the cases remain mild or asymptomatic, $15 \%$ are severe and 5\% infectious cases turn to critical, requiring ventilation.(2) Several clinical trials have been proposed for its treatment and management with supportive aim of mortality reduction.(1). By glancing a view on fig 1, it can be evidently seen that COVID-19 cases have started to rise significantly since last few months. Furthermore, as per World Health Organization (WHO), there have been 131,020,967 confirmed cases of COVID-19 at a global level recently.

COVID-19 shows a clear gender disparity in clinical outcome. Globally, infection rates between men and women are similar; yet epidemiological data revealed that men are more likely to have enhanced severity and mortality. In the current corona pandemic, as well as in both the 2003 SARS-CoV and the 2012 Middle East respiratory syndrome epidemics, females have substantially lower fatality rates than males as most of the immune regulatory genes are encoded by $\mathrm{X}$ chromosomes, resulting in women's generally stronger inflammatory response(2). This sex disparity in immune response is postulated to be largely driven by sex hormones.(3)

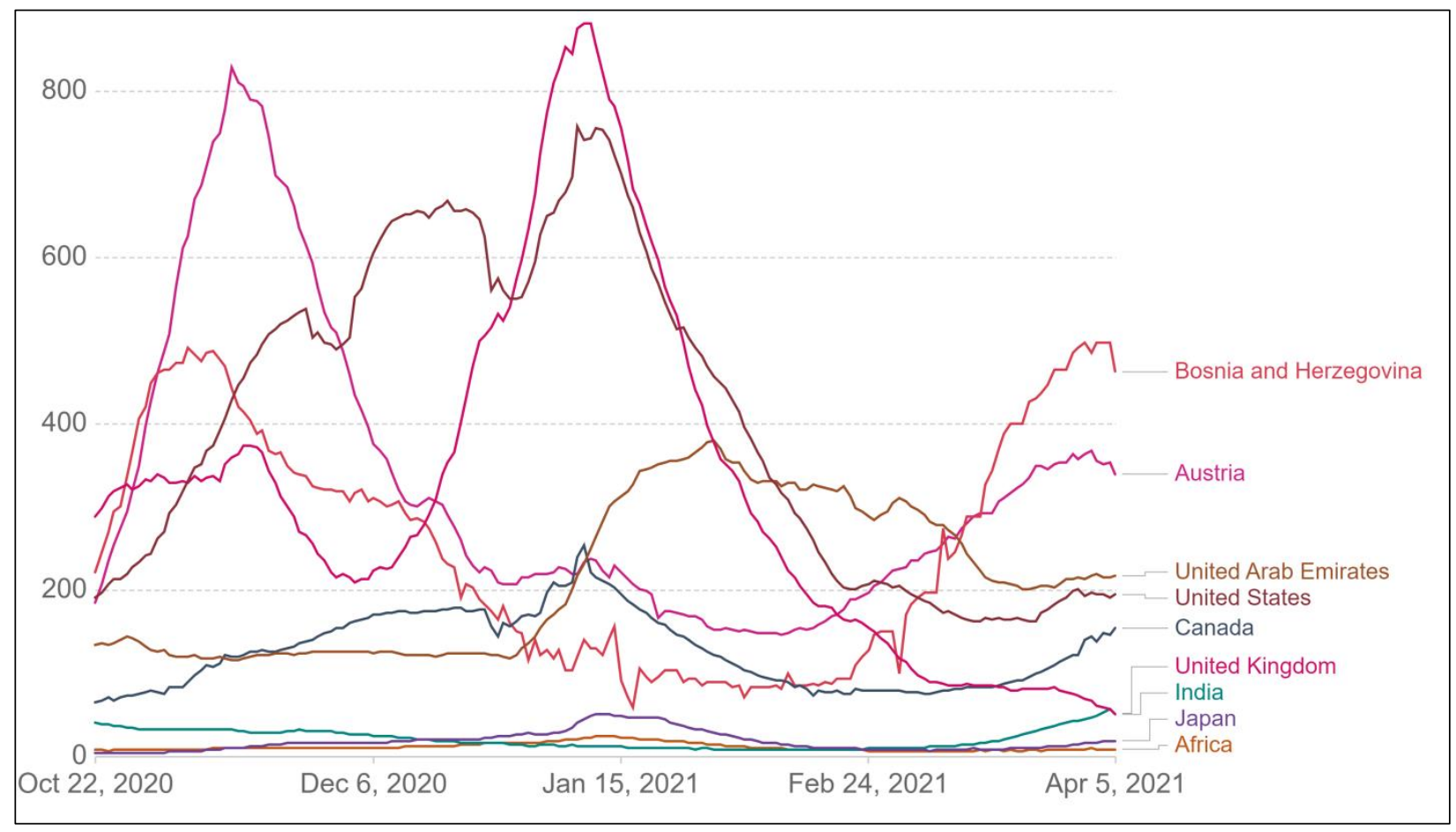

Fig 1: Comparison of rising COVID-19 cases between various countries (cases in per million people. (Source: https://ourworldindata.org/coronavirus) 


\section{Repurposed drugs for COVID-19}

The idea of repurposing drugs comes in to play when new diseases surface and there isn't enough time to develop new and effective therapies, such as in the case of Covid 19. Due to this reason a lot of therapeutic strategies for Covid 19 in the preliminary phase of the disease were based on repurposing other antiviral and immune drugs previously utilised for diseases such as HIV and MERS. These drugs can either affect the virus directly through RNA genome such as remedesvir which has been shown through trials to reduce viral infection, favipiravir which inhibits the enzyme RDRP, ribavirin which is a broad-spectrum antiviral drug having high EC50 of 109.50 $\mu \mathrm{M}$ and selectivity index more than 3.65 in Vero cells against SARS CoV2 (4).

Another category of repurposed drugs affects the virus through polypeptide packing such as protease inhibitors used for HIV (5) namely; lopinavir-ritonavir combination, darunavir which is a non polypeptide protease inhibitor.

A class of drugs which targets the host and improves immunity has also proved to be affective against the SARS CoV2. These include the interferons which are divided in to type I (IFN $\alpha$, IFN $\beta$, IFN $\omega$, and IFN $\tau$ ) and type II (IFN $\gamma$ ). They are directly involved with the innate immunity of the host and this therapy is effective against coronaviruses because they suppress the interferons of the host hence, replacing them is beneficial.

Drugs that target the virus uptake pathways such as chloroquine, arbidol and drugs that have antiinflammatory and immunomodulatory properties such as cepharanthine, JAK 2 inhibitors, tocilizumab, angiotensin receptor blockers and statins have also been repurposed for the treatment

of Covid 19. Some antiprotozoal agents such as nitazoxanide and ivermectin also show some antiviral properties and efficacy against coronaviruses.(5)

\section{Sex hormones: an alternative treatment of COVID-19}

It has been proved experimentally that the sex hormones estrogen and progesterone, are powerful immunomodulators at high concentrations and combination therapy with these two hormones may be used as a good therapeutic option to treat severe COVID-19 infection. Severity of infection and mortality associated with COVID-19 is comparatively lower in women than in men. This is because females develop a more effective immune response than males against infectious agents including viruses.(6) Differences in the immune and inflammatory responses due to hormonal differences between the two sexes, result in gender disparity observed in coronavirus vulnerability. (7) Estrogen has beneficial immunomodulatory and anti-inflammatory actions in mice and humans. It includes suppression of TNF- $\alpha$ and IL- 6 production by monocytes and macrophages. It also decreases IL-17 production and enhances regulatory $\mathrm{T}$ cells expression thus promoting immune tolerance.(6) Premarin is the only estrogen preparation which can induce beneficial effects in humans without significant adverse effects.

It is suggested that estrogen protects females against severe COVID-19 infection while androgen worsens COVID-19 outcome in males. Thus androgen deprivation therapy (ADT) may be considered as treatment option for severe infection.(7) Progesterone is another important 
immunomodulatory and anti-inflammatory hormone that inhibits IL-1 $\beta$ and IL-12 production by macrophages and dendritic cells. Progesterone treatment in experimental trials decreased the inflammatory reactions in the lungs, improved pulmonary function, and promoted pulmonary repair, which resulted in an earlier recovery. Acute hormone therapy with estrogen and progesterone in confirmed COVID-19 patients could mitigate the cytokine storm while increasing antibody production.(6)

\section{Conclusion and Future Aspects:}

Fatality rate due to COVID-19 is rapidly increasing with limited medical treatment modalities.(8) Many drugs like hydroxychloroquine, anti-viral agents like remdesivir and fevipiravir, steroids (dexamethasone), humanized antibodies, convalescent plasma and vaccination are available but none of the drug is completely curative in COVID-19.

Susceptibility and severity of COVID-19 shows sex based differences, affecting males more than females within the same age group. Such observations led various new studies to explore the safety and efficacy of sex hormones specifically estrogen and progesterone in COVID-19 treatment.9 The anti-inflammatory effect of estrogen against COVID is supported in several studies.(10) In a study on SARS-CoV1 infected mice, it was found that estrogen treatment decreases inflammatory reaction, virus titers, morbidity and mortality. Treatment with exogenous estrogen and testosterone inhibits the damaging inflammatory response to SARS-CoV-2 without hampering the immune system's response to the virus as corticosteroids do.(10)

Although the knowledge regarding sex hormones and COVID-19 is still in its infancy stage, however based on aforementioned discussions, it can be concluded that sex hormones could be as beneficial as potential treatment as potential treatment of COVID-19 in men and women in future. As the transgender community has been excluded from COVID-19 research studies all over the world, subsequently, this limits our understanding of how biological sex and the use of hormone modulators can impact the disease.(9) Further research is needed to explore the pharmacotherapeutic role of sex hormones and using them as the first line drugs in the treatment of COVID-19 disease. Moreover, there is a need to clearly understand the role of sex hormones in COVID-19 at molecular level. In addition, randomized clinical trials are highly recommended to evaluate safety, efficacy of sex hormone therapy in both men and women.

Acknowledgements: We are Thankful to the professors of the Universities and Our parents

Conflict of Interest: Authors don't have any COI

Funding: No Funding is Attached with this project

\section{REFERENCES:}

1.Li, H., Liu, S. M., Yu, X. H., Tang, S. L., \& Tang, C. K. (2020). Coronavirus disease 2019 (COVID-19): current status and future perspectives. International journal of antimicrobial agents, 55(5), 105951. https://doi.org/10.1016/j.ijantimicag.2020.105951 
2.Tsai, S. C., Lu, C. C., Bau, D. T., Chiu, Y. J., Yen, Y. T., Hsu, Y. M., Fu, C. W., Kuo, S. C., Lo, Y. S., Chiu, H. Y., Juan, Y. N., Tsai, F. J., \& Yang, J. S. (2021). Approaches towards fighting the COVID-19 pandemic (Review). International journal of molecular medicine, 47(1), 3-22. https://doi.org/10.3892/ijmm.2020.4794

3.Strope, J. D., Chau, C. H., \& Figg, W. D. (2020). Are sex discordant outcomes in COVID-19 related to sex hormones. Seminars in oncology, 47(5), 335-340. https://doi.org/10.1053/j.seminoncol.2020.06.002

4. Singh TU, Parida S, Lingaraju MC, Kesavan M, Kumar D, Singh RK. Drug repurposing approach to fight COVID-19. Pharmacological Reports. 2020 Sep 5:1-30.

5. Ngo ST, Quynh Anh Pham N, Thi Le L, Pham DH, Vu VV. Computational determination of potential inhibitors of SARS-CoV-2 main protease. Journal of chemical information and modeling. 2020 Jun 2.

6. Mauvais-Jarvis, F., Klein, S. L., \& Levin, E. R. (2020). Estradiol, Progesterone, Immunomodulation, and COVID-19 Outcomes. Endocrinology, 161(9), bqaa127. https://doi.org/10.1210/endocr/bqaa127

7. Acheampong, D. O., Barffour, I. K., Boye, A., Aninagyei, E., Ocansey, S., \& Morna, M. T. (2020). Male predisposition to severe COVID-19: Review of evidence and potential therapeutic prospects. Biomedicine \& pharmacotherapy = Biomedecine \& pharmacotherapie, 131, 110748. https://doi.org/10.1016/j.biopha.2020.110748

8. Trivedi, N., Verma, A., \& Kumar, D. (2020). Possible treatment and strategies for COVID-19: review and assessment. European review for medical and pharmacological sciences, 24(23), 12593-12608. https://doi.org/10.26355/eurrev_202012_24057

9. Wozniak, R. J., Nixon, D. F., \& Marston, J. L. (2020). Involvement of Cisgender and Transgender Individuals in Studies on the Impact of Hormonal Therapy on COVID-19. AIDS patient care and STDs, 34(9), 367-368. https://doi.org/10.1089/apc.2020.0118

10. Al-Lami, R. A., Urban, R. J., Volpi, E., Algburi, A., \& Baillargeon, J. (2020). Sex Hormones and Novel Corona Virus Infectious Disease (COVID-19). Mayo Clinic proceedings, 95(8), 17101714. https://doi.org/10.1016/j.mayocp.2020.05.013 